\title{
Assessing the Depth of the Subarachnoid Space by Ultrasound
}

\author{
Alexandre Gnaho ${ }^{1}$, Vinh Nguyen ${ }^{2}$, Thierry Villevielle ${ }^{2}$, Melina Frota ${ }^{3}$, Emmanuel Marret ${ }^{4}$, Marc E Gentili 5
}

Summary: Gnaho A, Nguyen V, Villevielle T, Frota M, Marret E, Gentili ME - Assessing the Depth of the Subarachnoid Space by Ultrasound.

Background and objectives: To assess the accuracy of the ultrasound (US) to predict the depth to reach lumbar intrathecal and epidural spaces in order to decrease the number of puncture attempts.

Methods: Thirty-one patients (25 males and 6 females), ASA I or II participated in this study. The transversal ultrasound image of the lumbar spine was obtained at the level of the L3-L4 space. An anesthesiologist without prior information performed the spinal anesthesia through the predicted target area. The distance between the skin and the anterior portion of the flavum ligamentum which is supposedly the bottom limit of the intrathecal depth or an approximation of the depth of the epidural space (ED-US) was measured by ultrasound and it was compared with the distance between the skin and the anterior portion of the flavum ligamentum on the needle (ED-N).

Results: ED-US and ED-N were respectively $5.15 \pm 0.95 \mathrm{~cm}$ and $5.14 \pm 0.97 \mathrm{~cm}$; these distances were not significantly different ( $p>0.0001)$. A significant correlation $r=0.982$ [95\% Cl 0.963-0.992, $p>0.0001$ ] was observed between the ED-US and ED-N measurements. Bland-Altman analysis showed an accuracy of $0.18 \mathrm{~cm}$; tolerated variations ranged from $-0.14 \mathrm{~cm}$ to $-0.58 \mathrm{~cm}$.

Conclusions: This study supports the idea that the US transversal plane allows the identification of axial anatomical structures and provides physicians with efficient information to perform spinal anesthesia.

Keywords: Ultrasonography, Interventional; Anesthesia, Spinal; Dura mater.

@2012 Elsevier Editora Ltda. All rights reserved.

\section{INTRODUCTION}

Ultrasound (US) guidance for regional anesthesia is a subject of major interest worldwide ${ }^{1}$. Since a few decades ago previous papers have been drawing the interest of physicians for the use of US for spinal or epidural anesthesia 2,3,4,5. Recently, US has been proposed as a preoperative assessment tool predicting the feasibility of neuroaxial blockade ${ }^{6}$. Nevertheless, physicians currently using US for neuroaxial anesthesia are still only a few, probably because this procedure requires not only a good knowledge of spinal sonoanatomy but also an advanced interventional skills. However, data related to the learning process for this technique are lacking. Spinal US of-

Received from Hôpital d'Instruction des Armées (HIA Begin), Saint Mandé, France.

1. MD; MBA; Department of Anesthesia and Intensive Care, HIA Begin

2. MD; Department of Anesthesia and Intensive Care, HIA Begin

3. MD; Anesthesiologist Department of Anesthesia and Intensive Care Centre Hospitalier Universitaire de Caen (CHU Caen)

4. MD; PhD; Professor of Anesthesiology. Department of Anesthesia and Intensive Care, Hôpital Universtitaire Tenon

5. MD; PhD; Professor of Anesthesiology. Department of Anesthesia and Intensive Care Centre Hospitalier Privé (CHP) Saint-Grégoire

Submitted on November 4, 2010.

Approved on March 21, 2011.

Correspondence to:

Alexandre Gnaho, MD, MBA

Department of Anesthesia and Intensive Care

Hôpital d'Instruction des Armées

Begin 94160 Saint Mandé, France

E-mail: agnaho@hotmail.fr fer valuable information to facilitate neuroaxial blockade. Most previous studies focused on the epidural space ${ }^{7,8}$. We believe that US images could also provide accurate estimation of depth to reach intrathecal space. This pilot study was designed to assess the reliability of US to predict lumbar intrathecal space depth and help to restrict the number of puncture attempts.

\section{METHODS}

\section{Participants}

Our Institutional Ethics Committee approved the study. Written informed consent was obtained from each patient. ASA I or II patients scheduled for lower extremity surgery were enrolled. Patients with spinal deformities or previous spinal surgery were excluded. Data collection was planned as a cohort study.

\section{Measurements and puncture methods}

The palpation of bony surface landmarks was done by one investigator before location and rated as good (very well palpable), moderate (well palpable), or none (hardly or not palpable). Images were obtained with patients in the sitting position. For selection of L3-L4 interspace, a linear array probe 5-10 MHz (TITAN: Sonosite Inc TM, Bothell, WA, USA) was 
placed by another investigator over the sacrum two or three centimeters to the right of the midline to visualize the hyperechoic line corresponding to the image of the sacrum.

The probe was then moved cephalad to obtain the classic hyperechoic saw-like image representing the articular processes, and the interspaces were counted upward till the L3L4 interspace. At this time, a transverse scan was performed. The midline was identified using the after shadow of the spine, and the L3-L4 interspace was identified by moving the probe slowly cephalad or caudad in order to obtain the absence of after shadow and the view of the complex formed by the ligamentum flavum-dura mater and also to find the vertebral body. However, our equipment does not always allow us to visualize ligamentum flavum and dura mater as two separate structures but often as only one hyperechoic line. At this time the optimum sonogram, depicted according to an agreement between two investigators, was frozen. The outlines of the probe and the target in its center corresponding to the puncture point was drawn with a dermographic pen.

Two investigators analyzed the images, and the US visibility of anatomical structures (corpora vertebrae, thecal sac, and ligamentum flavum-dura mater complex) was rated as good (very well defined), moderate (well defined), or none (hardly or not defined). We recorded the time necessary to obtain an optimum sonogram of the structures (location time), which also included the time elapsed for making the outlines of the probe and the target point. We measured with a built-in caliper the distance from the skin to the anterior part of the ligamentum flavum-dura mater complex (ED), which is supposed to be the bottom limit before reaching intrathecal space or an approximation of the epidural space depth (ED-US). Subsequently, a blinded anesthesiologist to this measure performed spinal anesthesia (25 G pencil spinal needle, B. Braun, Melsungen AG, Germany) through the predicted target point. The measurement of the distance from skin to the anterior part of the ligamentum flavum-dura mater complex was obtained after marking the needle with a sterile skin-marking pen (ED-N). The technique: one hand of the anesthesiologist held firmly the needle and the point of measure was pinched between the thumb and the index of the other hand to secure measurement. ED-US was compared with ED-N. During puncture, each advancement of needle was considered as puncture attempt. If spinal anesthesia was not possible at L3-L4 interspace, the anesthesiologist punctured a lower vertebral interspace. The number of attempts was recorded.

\section{Statistical methods}

Data were summarized as mean \pm standard deviation (SD). ED was calculated from ultrasound device (ED-US) and directly during needle punctures (ED-N). Correlation between ED-US and ED-N was determined by simple linear regression analysis using the least-squares method. We constructed BlandAltman plots of differences to averages of the epidural depth measured by the two methods and calculated the bias, precision (1 SD), and $95 \%$ limits of agreement (mean bias $\pm S D$ ).
Excel Microsoft (Chicago, IL) and Medcalc software were used for statistical analysis. Two-sided $p$ value $<0.05$ was considered statistically significant.

\section{RESULTS}

Thirty one patients underwent spinal anesthesia for lower extremity surgery (25 males and 6 females). The average age was $43 \pm 15 \mathrm{yr}$, weight $79 \pm 14 \mathrm{~kg}$, length $174 \pm 8 \mathrm{~cm}$, and body mass index $27 \pm 3 \mathrm{~kg} \cdot \mathrm{m}^{-2}$. The visibility of anatomical structures was good in $87 \%$ of patients and moderate in $13 \%$ of patients (Figures $1 \mathrm{~A}$ and $1 \mathrm{~B}$ ). The location time was $76 \pm 18$ seconds. The ED-US and ED-N were $5.15 \pm 0.95 \mathrm{~cm}$ and $5.14 \pm 0.97 \mathrm{~cm}$, respectively. These distances were not significantly different $(p>0.0001)$. In addition, a significant correlation $r=0.982$, [95\% Cl 0.963-0.992, $p>0.0001$ ] was observed between ED-US and ED-N measurements (Figure 2). Bland-Altman analysis between these two measures is shown in Figure 3. The mean difference (bias) was $0.22 \mathrm{~cm}$, with a precision of $0.18 \mathrm{~cm}[95 \% \mathrm{Cl} 0.15-0.29]$. The limits of agreement were $-0.14 \mathrm{~cm}[95 \% \mathrm{Cl}(-0.25 ;-0.01)]$ to $-0.58 \mathrm{~cm}$ $[95 \% \mathrm{Cl}(-0.46 ;-0.70)]$.
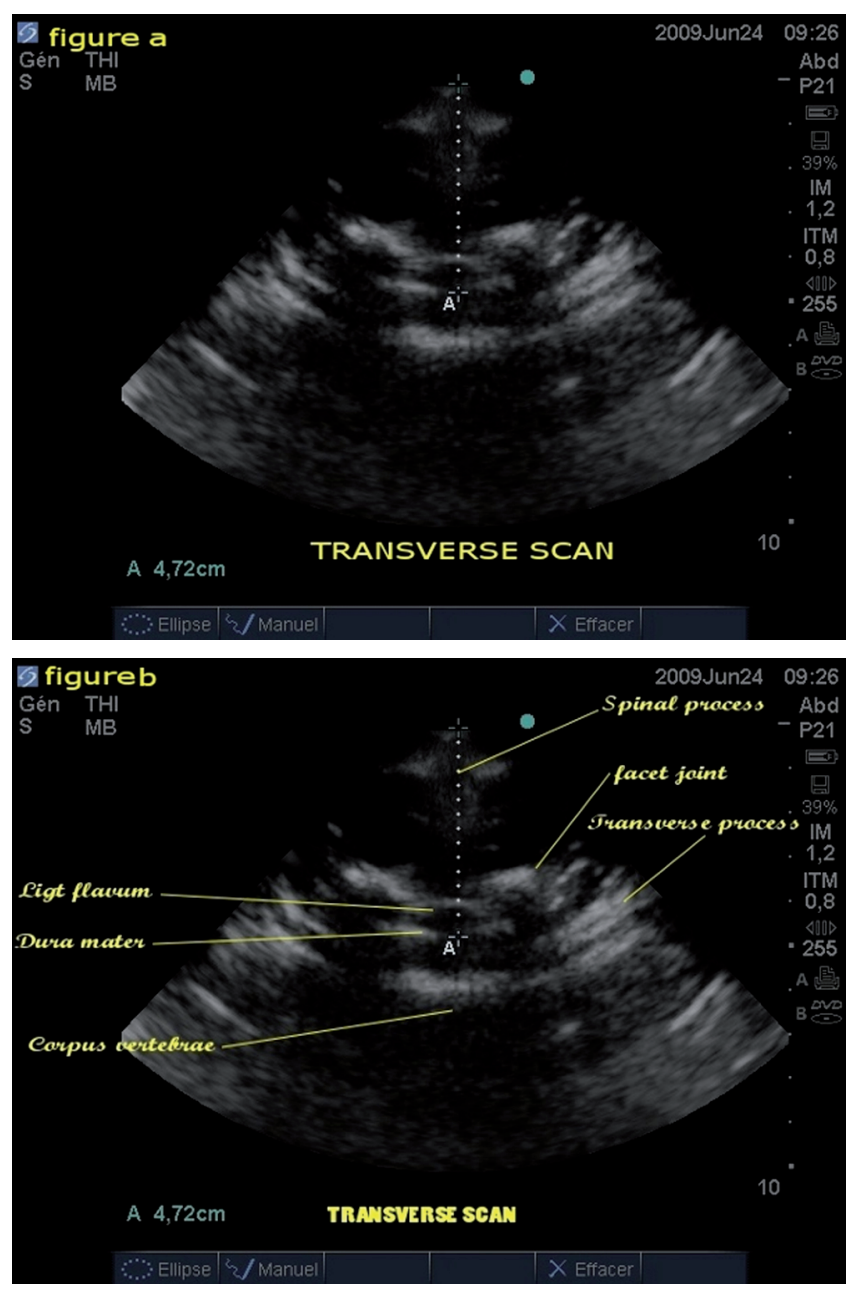

Figure $1 \mathrm{~A}$ and $1 \mathrm{~B}$ 


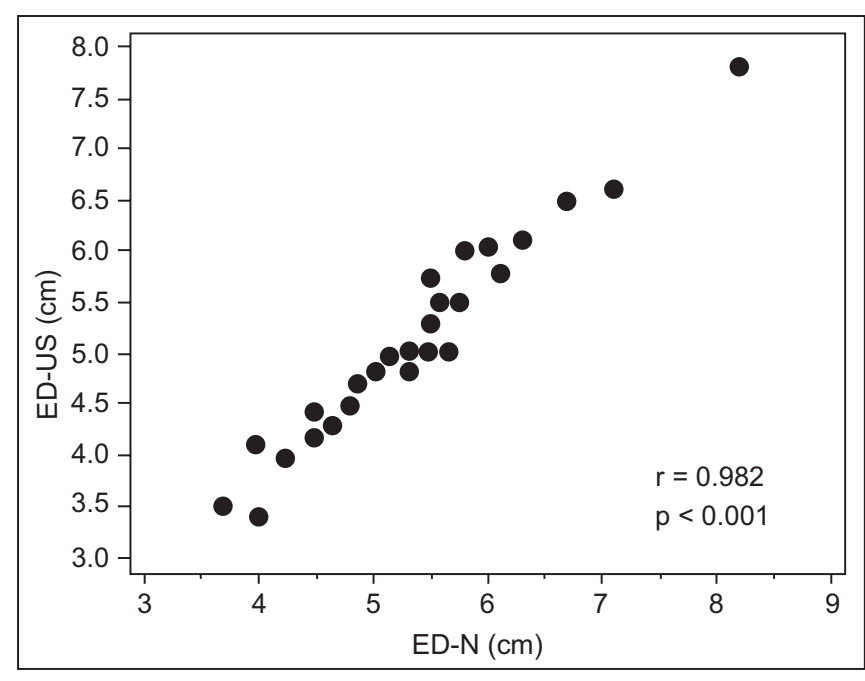

Figura 2 - Correlation between US Depth (ED-US) in $\mathrm{cm}$ and Needle Depth (ED-N) in cm.

Spinal anesthesia was performed at first attempt with 24 patients $(78 \%), 2$ attempts were necessary for 5 patients (16\%), and one patient (3\%) needed 4 attempts. Dural access was impossible for another patient (3\%). Bony surface landmarks were absent before location in $68 \%$ of patients. Visibility of anatomical structures was good in $87 \%$ of patients and moderate for the remaining participantes.

\section{DISCUSSION}

This study supports the condition that US transverse plane is likely to identify axial anatomical structures and may provide efficient information to practitioners to perform spinal anesthesia.

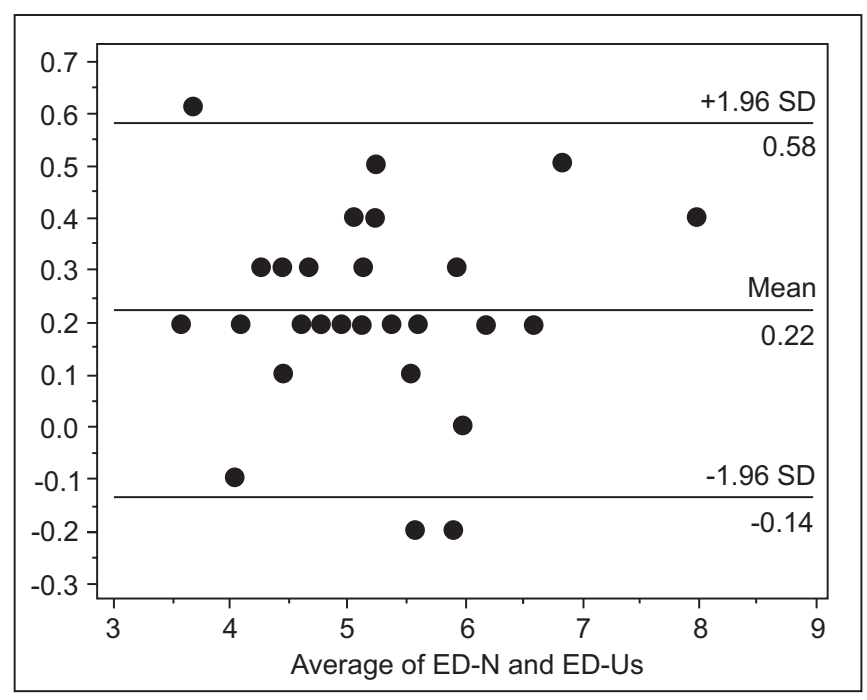

Figura 3 - Bland-Altman Analysis: Agreement between Ultrasound Depth (ED-US) and Needle Depth (ED-N).

\section{Comparison with previous studies}

Previous studies in obese and nonobese patients have demonstrated a good correlation between ED-US and ED-N: data are summarized in Table I.

At this time, US guidance was used to improve epidural approach in 76 surgical patients ${ }^{2,9}$; in 76 parturients undergoing caesarean section under epidural anesthesia 10,11; and overall in 427 parturients receiving epidural analgesia for delivery 13-15. However most previous studies did not focus on spinal anesthesia, but epidural anesthesia. The method of indirect US guidance allows identification in a midline sagittal scan of lumbar vertebrae and an accurate measurement of the depth

Table I - Overview on Correlation and Precision of US-assisted Epidural Puncture

\begin{tabular}{|c|c|c|c|c|c|}
\hline Studies & Patients & Number & Depth $(\mathrm{mm})$ & Correlation & Bland-coefficient Altman* \\
\hline Cork et al. ${ }^{2}$ & $\mathrm{Ea}$ & 36 & 45.0 & 0.98 & No data \\
\hline Currie ${ }^{13}$ & Ea in obstetric & 75 & $41.2 \pm 8.1$ & 0.92 & 5.4 \\
\hline Wallace et al. ${ }^{10}$ & Ea cesarian section & 36 & $55.1 \pm 2.1$ & 0.98 & 5.4 \\
\hline Bonazzi et al. ${ }^{9}$ & Herniotome & 40 & $51.0 \pm 6.2$ & 0.98 & No data \\
\hline Grau et al. ${ }^{15}$ & Ea in obstetric & 100 & $53.0 \pm 7.0$ & 0.79 & 6.8 \\
\hline Grau et al. ${ }^{3}$ & CSE cesarian section & 80 & $51.5 \pm 9.3$ & 0.92 & 5.1 \\
\hline Grau et al. ${ }^{4}$ & Ea in obstetric & 300 & $51.2 \pm 7.0$ & 0.83 & 6.9 \\
\hline Arzola et al. ${ }^{19}$ & Ea in obstetric & 61 & $46.6 \pm 6.8$ & 0.88 & 6.6 \\
\hline Lee et al ${ }^{12}$ & Ea in obstetric & 36 & $43.8 \pm 5.1$ & No data & No data \\
\hline Balki et al. ${ }^{20}$ & Ea obese in obstetric & 46 & $63.0 \pm 8.0$ & 0.85 & No data \\
\hline Tran et al. ${ }^{18}$ & Ea in obstetric & 20 & $51.0 \pm 11.0$ & 0.80 & No data \\
\hline
\end{tabular}

* Bland and Altman analysis of the precision between US measures of the epidural depth and the depth estimated from needle measures. Ea: Epidural anesthesia; CSE: combined epidural and spinal anesthesia. 
to reach intrathecal space. This procedure can facilitate the performance for spinal anesthesia and may decrease the rate of complications, most probably in those patients whose anatomic landmarks are obscured 9,10,16,17.

US imaging may reduce attempts to lumbar approach, considering that prior to it palpation was the only way to lumbar puncture approach. A significant reduction of number puncture has been demonstrated when performing combined spinal and epidural anesthesia ${ }^{3}$. In addition, the same authors have shown the value of pre-puncture sonographic informations for lumbar access in obstetric field ${ }^{4}$. Grau et al. ${ }^{5}$ have shown changes that occur on spinal and epidural anatomy during pregnancy using US.

Our study reinforces the usefulness of pre-puncture information such as optimum puncture point, depth to reach intrathecal space, and the visibility of neuroaxial anatomical structures. In some patients, performing spinal anesthesia is challenging. However, the presence of these healthy young patients in which bony surface landmarks were not palpable in our study was an unexpected observation. Assessment of palpation upon agreement of two investigators instead of one should have influenced this rate. Nevertheless, this surprising observation did not affect the study results.

In 60 participants (20 parturients and 40 healthy volunteers in general surgery), Grau et al. ${ }^{5}$ have shown that longitudinal paramedian access provides a larger permeable window, and improves the quality of pre-puncture diagnostics for neuroaxial anesthesia or analgesia ${ }^{18}$. Despite this classic superiority of the longitudinal paramedian access to provide better sonograms of lumbar spine, we have been able to identify neuroaxial structures using transverse median plane. Such approach seems to be closer to the reality of our clinical practice. Some recent studies focus on transverse plane providing reliable landmarks for epidural anesthesia ${ }^{19,20}$. In some cases such as impalpable bony landmarks, several unsuccessfuly puncture attempts, patient becoming irritable etc., when things do not go as smoothly like in the textbook, transverse and paramedian longitudinal approaches could be used in a complementary way ${ }^{21}$. In addition, to our knowledge, there is no randomized and prospective study comparing longitudinal and transverse approaches for spinal anesthesia. Furthermore, we did not encounter significantly calcifications on scanning area; probably because of our young population. The reflexion involved in these calcifications are often described in transverse scanning plane and is supposed to further difficult lumbar access, particularly in elderly patients.

ED-US measurement must be expected to differ slightly from the ED-N measurement. This degree of uncertainty could be erased by a real time monitoring of the lumbar puncture 22 .

We used a linear 5-10 MHZ probe, while in most papers the transducer often used was a sector 2-5 MHZ probe for spinal ultrasound imaging, mainly because top managers of our institution were in a context of difficult financial choice to make. The result was that we could not acquire a complete US device. So the sector 2-5 MHZ probe was not available. In addition, Ferre et al. ${ }^{23}$ had been able to identify neuroaxial structure with a linear array probe with a good delineation of these structures in $93.4 \%$ of patients.

\section{Clinical implications}

Several reports suggest the use of US to perform neuroxial blockade in difficult anatomical situations, such as obesity, scoliosis, or edema. Nevertheless, training is probably needed in easier clinical presentations as to acquire proficiency in order to perform later a successfully spinal anesthesia that could be difficult or impossible without sonographic landmarks.

In a randomized trial evaluating epidural access in 72 patients with difficult history of epidural anesthesia and/or substantial alterations of lumbar spine (such as scoliosis, kyphosis, hyperlordosis and BMI $\left.>33 \mathrm{~kg} \cdot \mathrm{m}^{-2}\right)$, Grau et al. ${ }^{5}$ have demonstrated in the US group (36 patients) a higher satisfaction, an improved visual analogical scale of pain score, and fewer puncture attempts ${ }^{11}$. The advantages included a target definition facilitating lumbar puncture, direct visualization of all neuroaxial structures with fewer bone contacts and thus reducing complications and side effects. Lumbar spine ultrasound could be a useful clinical tool to facilitate needle insertion. Lee et al have shown that ultrasonography makes it possible to detect abnormal sonoanatomy. ${ }^{12}$ We can decrease complications such as unintentional dural punctures.

Pediatric anesthesia is the other field in which spinal or epidural blockade is challenging. The exclusive technique of loss of resistance usually performed with a child under general anesthesia has been associated with complications and adverse outcomes, including more significantly neurological deficit as a result of unintentional spinal cord trauma ${ }^{24,25}$. In a prospective randomized study of 64 children evaluating the feasibility, number of bone contacts, and duration for the performance of epidural anesthesia using US guidance compared with the loss of resistance method, Willschke et al. ${ }^{26}$ have shown that direct visualization of intra-epidural spread of local anesthetic was a reliable way to verify position of epidural catheter. In addition, they have demonstrated that US guidance in experienced hands reduces both the risk of bone contact as well as duration for catheter placement.

\section{Potential limitations}

Sophisticated and costly equipment and sometimes difficulties for scan performance may be considered as some few disadvantages. The availability of a complete machine in the institution is also a problem. Therefore, ultrasound device is commonly used for other professional approaches like venous catheterism or hemodynamic assessments. US anat- 
omy knowledge and training are strongly necessary to use this technology. Interpretation of images may be also difficult some times. In addition, the acoustic windows for US spine are very limited ${ }^{15}$. This is part of the explanation why US imaging for neuroaxial anesthesia had been such a poor source of interest for decades.
In conclusion, US makes it possible to have an accurate estimation of the depth to reach intrathecal space, we also had a good delineation of neuroaxial structures despite the limitations of our ultrasound device. Further randomized trials are required to establish the role of US for spinal anesthesia in clinical practice. 


\section{REFERENCES}

1. Gray AT - Ultrasound-guided regional anesthesia: current state of the art. Anesthesiology, 2006;104:368-373.

2. Cork RC, Kryc JJ, Vaughan RW - Ultrasonic localization of the lumbar epidural space. Anesthesiology, 1980;52:513-516.

3. Grau T, Leipold RW, Conradi R, Martin E, Motsch J. Ultrasound facilitates localization of the epidural space during combined spinal and epidural anesthesia. Reg Anesth Pain Med. 2001; 26:64-67

4. Grau T, Leipold RW, Conradi R, Martin E, Motsch J.Efficacy of ultrasound imaging in obstetric epidural anesthesia. J Clin Anesth 2002; 14:169-75

5. Grau T, Leipold RW, Horter J et al. -The lumbar epidural space in pregnancy: visualization by ultrasonography. $\mathrm{Br} \mathrm{J}$ Anaesth, 2001;86:798804.

6. Chin $\mathrm{KJ}$, Chan V - Ultrasonography as a preoperative assessment tool: predicting the feasibility of central neuraxial blockade. Anesth Analg, 2010;110:252-3.

7. Helavel PE, Conceiçao DB, Swarovsky C et al. - Evaluating the depth of the epidural space with the use of ultrasound. Rev Bras Anestesiol, 2010;60:376-82.

8. Schlotterbeck H, Schaeffer R Dow WA et al. - Ultrasonographic control of the puncture level for lumbar neuraxial block in obstetric anaesthesia. Br J Anaesth, 2008;100:230-234.

9. Bonazzi M, Bianchi De Grazia L, Di Gennaro S et al. - Ultrasonography-guided identification of the lumbar epidural space. Minerva Anesthesiol, 1995;61:201-205.

10. Wallace DH, Currie JM, Gilstrap LC et al. - Indirect sonographic guidance for epidural anesthesia in obese pregnant patients. Reg Anesth, $1992 ; 17: 233-236$.

11. Grau T, Leipold W, Conrandi R et al. - Ultrasound control for presumed difficult epidural puncture. Acta Anaesthesiol Scand, 2001;45:766771.

12. Lee $Y$, Tanaka M, Carvalho JCA. Sonoanatomy of the lumbar spine in patients with previous unintentional dural punctures during labor epidurals. 2008; 33: 266-270

13. Currie JM - Measurement of the depth to the extradural space using ultrasound. Br J Anaesth, 1984;56:345-347.

14. Grau T, Leipold RW, Conrandi R et al. - Paramedian access to the epidural space: the optimum window for ultrasound imaging. J Clin Anesth, 2001;13:213-217.

15. Grau T, Leipold R, Conradi R et al. - Ultrasonography and peridural anesthesia. Technical possibilities and limitations of ultrasonic examination of the epidural space. Anaesthesist, 2001;50:94-101.

16. O'Donnell D, Prasad A, Perlas A - Ultrasound-assisted spinal anesthesia in obese patients. Can J Anaesth, 2009;56:982-983.

17. Prasad GA, Tumber PS, Lupu CM - Ultrasound guided spinal anesthesia. Can J Anaesth, 2008;55:716-717.

18. Tran D, Kamani AA, Lessoway VA et al. - Preinsertion paramedian ultrasound guidance for epidural anesthesia. Anesth Analg, 2009;109:661-667.

19. Arzola C, Davies S, Rofaeel A et al. - Ultrasound using transverse approach to the lumbar spine provides reliable landmarks for labor epidurals. Anesth Analg, 2007;104:1188-1192.

20. Balki M, Lee $Y$, Halpern $S$ et al. - Ultrasound imaging of the lumbar spine in the transverse plane: the correlation between estimated and actual depth to the epidural space in obese parturients. Anesth Analg, 2009;108:1876-1881.

21. O'Donnell D, Prasad A, Perlas A - Ultrasound assisted spinal anesthesia in obese patient. Can J Anesth, 2009;56:982-983.

22. Karmakar MK, Li X, Ho AMH et al. - Real time ultrasound-guided paramedian access evaluation of a novel in plane technique. Br J Anaesth, 2009;120:845-854.

23. Ferre RM, Sweeney TW - Emergency physicians can easily obtain ultrasound images of anatomical landmarks relevant to lumbar puncture. J Emerg Med, 2007;25:291-296.

24. Flandin -Bletry C, Barrier G - Accidents following extradural analgesia in children. A result of a retrospective study. Paediatr Anaesth, 1995;5:41-46.

25. Rose JB - Spinal cord injury in a child after single shot epidural anesthesia. Anesth Analg, 2003;96:3-6.

26. Willschke $H$, Marhofer $P$, Bösenberg A et al. - Epidural catheter placement in children: comparing a novel approach using ultrasound guidance and standard loss of resistance technique. $\mathrm{Br} \mathrm{J}$ Anaesth, 2006;97:200-207. 